www.nature.com/pj

\title{
Application of catastrophe theory to neck initiation of metallocene-catalyzed high-density polyethylene
}

\author{
Koh-hei Nitta and Mizue Kuriyagawa
}

This paper examines the initiation of necking in a metallocene-catalyzed high-density polyethylene. When the specimen is uniaxially deformed, the necking is usually initiated between the first and second yield points, and the neck is stabilized at the second yield point. The initiation of necking was explained by a first-order catastrophic phase transition analogous to the model of a van der Waals gas. At the first yield point, the stacked lamellar clusters are fragmented into cubic cluster units of single chain size; the rearrangement of these units under uniaxial tension produces the texture of necking. Catastrophic arrangement of the cluster units results in a sudden emergence of a locally close-packed layer structure. This is the precursor of necking, and several layered plates are stabilized at the second yield point. The layered plate collapses via sliding of the cubic units by means of the interchain links, to form macroscopic neck; the plate thus behaves as a unit for the necking process. The continual collapse from one plate to another corresponds to the neck propagation.

Polymer Journal (2012) 44, 245-251; doi:10.1038/pj.2011.119; published online 23 November 2011

Keywords: catastrophe theory; cold drawing; neck initiation; polyethylene

\section{INTRODUCTION}

The uniaxial extension for most semicrystalline polymer solids shows stepwise behavior as shown in Figure 1, where a distinct yield point appears as a maximum in the conventional stress-strain curve and is the onset point of temporary plastic deformation. ${ }^{1}$ Beyond the yield point, a concave contraction suddenly initiates on the specimen and coalesces into a well-defined neck by around the second yield point; the boundary of the neck propagates under constant flow stress. The second yield point is the onset of the permanent plastic deformation. ${ }^{1}$ This necking is called 'cold drawing'2 and this paper focuses on the cold-drawing process.

The second yield point is more clearly observed in the case of polyethylene-based materials, but this double yield point seems to be a general feature of semicrystalline polymers as suggested by Séguéla et $a .^{3}$ In the necking process, the macroscopic morphological transformation from isotropic spherulitic to anisotropic fibril structures takes place accompanied by the destruction and/or rearrangement of the parts of the stacked crystalline lamellae. The extension ratio in the neck propagation region is generally defined as the natural draw ratio. ${ }^{4,5}$ Finally, after the necking boundaries have propagated throughout the entire length of the specimen, the deformation curve exhibits strain-hardening behavior, and the specimen breaks.

Previously, we have proposed a structural model for the yielding mechanism, where the yielding is caused by fragmentation of stacked lamellar clusters. ${ }^{6}$ According to the lamellar cluster theory, a lamellar clustering process occurs during the supercooling of polyethylene (PE) chains and some extended molecules longitudinally bind to at most two adjacent cluster units. These extended molecules bridge two adjacent units and act as stress transmitters. The bending force applied through the intercluster link, which is composed of several active tie molecules bridging between clusters, decomposes the lamellar clusters into their fragments (cluster units) at the yield point. Consequently, each side of cluster units is comparable to the end-to-end distance of chains.

The above view has been supported indirectly by mechanical data and directly by electron microscopy. ${ }^{8,9}$ On the basis of the lamellar cluster model, we found a simple structural model for predicting the values of the natural draw ratio using a series of metallocene-catalyzed high-density polyethylenes (HDPEs). ${ }^{10}$ The electron microscopic images of the necked portion showed the appearance of granular units at the shoulder region of the necked specimen. The granular units correspond to the lamellar cluster units fragmented from the lamellar clusters at the yield point. The cluster units are microscopically close packed and then collapse into cuboidal objects in such a way as to minimize the total free energy. Consequently, the theoretical model explained the fact that the natural draw ratio decreases with increasing the weight-average molecular weight ${ }^{1}$ and is dependent on the dimensions of the specimen cross-section, for example, the natural draw ratio is a maximum when the shape of the cross-section is square.

The aim of the current work is to explore the initiation and formation of necking under tensile deformation. It is well known that tensile deformation of a sample is homogeneous up to the first yield point, whereas once a neck initiates, the deformation is inhomogeneous, and macroscopic highly oriented regions appear in the specimen. ${ }^{1,11}$ Hutchingson et al. ${ }^{12}$ demonstrated that the tensile 


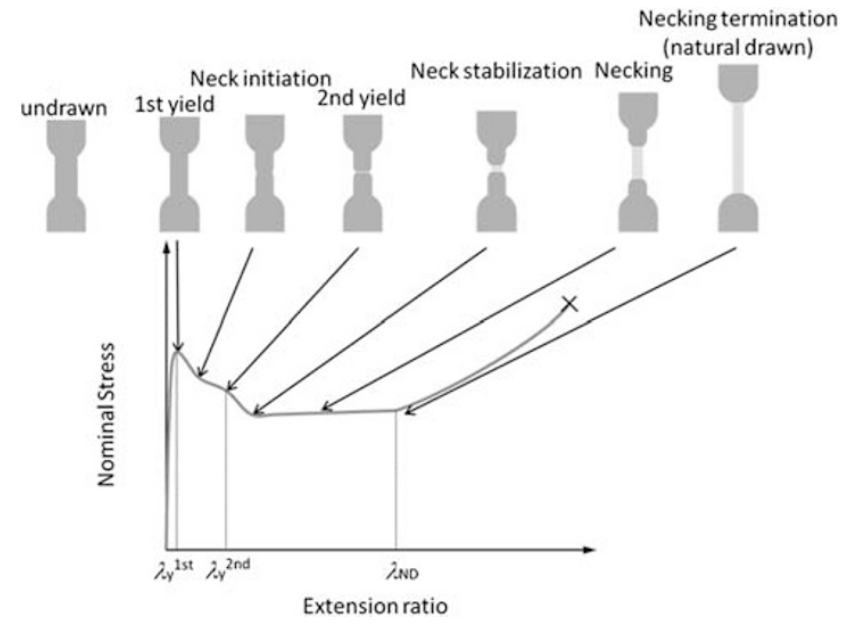

Figure 1 Relationship between tensile stress-extension ratio curve and shape of the specimen. $\lambda_{y}{ }^{1 s t}$ is the first yield ratio, $\lambda_{y}{ }^{2 n d}$ is the second yield ratio and $\lambda_{N D}$ is the natural draw ratio.

necking process can be treated as a first-order phase transition induced by force. Erickson ${ }^{13}$ showed that a remarkable analogy can be made between the necking process of a forced elastic bar and a phase transition in a van der Waals gas. Kilian et al. ${ }^{14,15}$ showed that the constitutive relation of semicrystalline polymers can be expressed by the extension of the ideal gas model to the van der Waals equation where bulky fragmented lamellar clusters are assumed to behave as junction points. ${ }^{16}$ Based on these excellent theoretical considerations, Nitta-Takayanagi ${ }^{17}$ explained the initiation of a neck by the mathematical fact that the van der Waals equation shows a first-order catastrophic phase transition. In this work, we apply the catastrophe theory to the initiation of necking in a metallocene-catalyzed HDPE.

\section{EXPERIMENTAL PROCEDURE}

\section{Materials and sample preparation}

We employed a metallocene-catalyzed HDPE with weight-average molecular weight of $96 \times 10^{3}$ and molecular weight distribution index of 2.5. The HDPE pellets were compression molded in a laboratory hot press for $5 \mathrm{~min}$ at $210^{\circ} \mathrm{C}$ and $10 \mathrm{MPa}$, and then quenched in iced water to give sample sheets with thickness about $0.3 \mathrm{~mm}$. These sheet samples were annealed in an oven at $100^{\circ} \mathrm{C}$ for $5 \mathrm{~h}$, to obtain sheets with thermodynamically stable structures.

\section{Characterization}

The crystallinity was $61 \mathrm{vol} . \%$, as estimated from the Archimedes method using a Mettler electrobalance XS205 (Mettler, Greifensee, Switzerland), with ethanol as the solvent, and taking the densities of the crystalline and amorphous regions as 1000 and $856 \mathrm{~kg} \mathrm{~m}^{-3}$, respectively.

The melting temperature measured by differential scanning calorimetry (using a Perkin Elmer Diamond DSC at a heating rate of $20^{\circ} \mathrm{C} \mathrm{min}^{-1}$ ) (Perkin Elmer, Winter Street Waltham, MA, USA) was $133^{\circ} \mathrm{C}$. The long period estimated from the small angle X-ray scattering peak was $27.5 \mathrm{~nm}$. The small angle X-ray scattering measurements were performed on the 10C beam line in the Photon Factory, Tsukuba, Japan. Dynamic mechanical spectra were measured from $-150{ }^{\circ} \mathrm{C}$ to $140{ }^{\circ} \mathrm{C}$ at a constant frequency of $10 \mathrm{~Hz}$ and at a heating rate of $2^{\circ} \mathrm{C} \mathrm{min}^{-1}$ using a dynamic mechanical analyzer UBM DVE-V4 (UBM, Kyoto, Japan). Two typical relaxation peaks were observed, identified conventionally as $\alpha$ (overlapping $\alpha_{1}$ and $\alpha_{2}$ ) and $\gamma \cdot{ }^{18}$ The $\gamma$ relaxation peak, associated with the glass transition or local motion of the amorphous phases, was at about $-120^{\circ} \mathrm{C}$. The $\alpha_{1}$ process, associated with slip processes of crystal blocks and crystallites (or grain boundary phenomena) and the $\alpha_{2}$ process, associated with intracrystalline chain motion or quasi melting of the crystal lattice, were observed at 63 and $123^{\circ} \mathrm{C}$, respectively.

\section{Tensile tests}

To investigate the temperature dependence of the necking behavior, we performed tensile tests at fixed elongation speeds ranging from 0.10 to $0.65 \mathrm{~mm} \mathrm{~s}^{-1}$, at temperatures from 25 to $130^{\circ} \mathrm{C}$ using an Instron tensile tester Model 4466 (Instron Japan, Kawasaki, Japan). Dumbbell-shaped specimens with width $4 \mathrm{~mm}$ and gauge length $10 \mathrm{~mm}$ were used. These specimens were elongated up to a chosen extension ratio of 4.4, which is in the necking region, and the necked specimen was held for $10 \mathrm{~min}$. Then the necked specimens were released from the clamps and stored for 1 day at room temperature. The values of natural draw ratio were calculated as the initial area divided by the necked area.

\section{THEORY}

As shown in the previous paper, ${ }^{18}$ the stress of HDPE, post first yielding, over the range of strain rates and temperatures involved in typical tensile experiments can be expressed by an additive equation of the plastic and the elastic components. ${ }^{19-21}$

$$
\sigma=\sigma_{p}(\lambda, \dot{\lambda}, T)+\sigma_{e}(\lambda, T)
$$

where $\lambda$ is the extension ratio, $\dot{\lambda}$ is the time derivative of $\lambda$ and $T$ is the absolute temperature. These are nominal stresses. The plastic component can be assumed to be expressed by an Eyring process, ${ }^{22}$ and can be given by

$$
\sigma_{p}(\lambda, \dot{\lambda}, T)=\frac{\Delta H}{V_{F}}\left(1-T / T_{p}\right)
$$

where

$$
T_{p}=\frac{\Delta H}{R}\left(\frac{\ln \dot{\varepsilon}_{0}}{\dot{\varepsilon}}\right)^{-1}
$$

$V_{F}$ is the activation volume of sliding units, $R$ is the gas constant, $\Delta H$ is the activation energy for plastic flow, $\dot{\varepsilon}_{0}$ is the constant pre-exponential factor and $\dot{\varepsilon}$ is the strain rate. Therefore, it was predicted that the plastic flow stress linearly decreases to zero as the temperature approached a temperature $T_{p}$ and that the inverse of $T_{p}$ is proportional to $-\ln \dot{\varepsilon}$.

Nitta-Takayanagi ${ }^{6}$ have succeeded in explaining the emergence of intercrystalline links (ICLs) and piles of crystalline lamellae within PE spherulites, as shown in the electron microscopic pictures by Keith et al. ${ }^{8}$ According to the theoretical consideration of the yielding process, there are two types of tie chains: inactive tie chains, which bind several stacked lamellae to form lamellar clusters; and active tie chains, which bridge two adjacent lamellar clusters. ${ }^{23}$ The ICLs are bundles of active tie chains, which support the external force. It should be noted here that the extended tie links longitudinally connect at most two adjacent units owing to the limitation of chain extension. Thus, their tractive forces alternately act on the cluster surface, leading a portion of the lamellar cluster to be bent around one ICL. The fragmentation of lamellar clusters releases the ICLs embedded in the two adjacent lamellar clusters and makes it possible to cause rearrangement of the ICL units decorated with the cluster units. Therefore, the decorated dumbbell-shaped ICL units, or micro shish-kebab structures, randomly disperse in the spherulitic matrix just after the first yield point, and yielding deformation leads to localized ordering of the ICL units (attractive interaction), while the volume excluded effects of the cluster units exert a repulsive interaction. These structural features of ICL units in the neck region are strongly supported by the transmission electron microscope (TEM) results of Kanig $^{24}$ who found that a network of fibers cross-linked by crystal blocks is formed on deformed PE films. Kilian et al. ${ }^{14-16}$ also identified stacks of several lamellae running parallel to one another and forming clusters, on the basis of TEM observations of a uniaxially deformed PE. Moreover, they showed that the stress-strain curves of a molecular 
network model with bulky junction points can be described by a van der Waals type of equation, ${ }^{15}$ and applied this equation to the stressstrain curves of some PE materials. ${ }^{14}$ The existence of network structure formed during crystallization of the isotropic PE samples has also been shown experimentally in the textbook by Strobl. ${ }^{25}$

The ideal gas model of G'Sell et al., ${ }^{19}$ which had been used for network component $\sigma_{e}$, was replaced by Kilian's van der Waals model:

$$
\sigma_{e}(\lambda, T)=\frac{N k T e}{1-b e}-a e^{2}
$$

where $k$ is the Boltzmann constant, and the extent of deformation $e=\lambda-\lambda^{-2}$, which corresponds to the inverse of the gas volume in the equation of state. ${ }^{26}$ The parameter $b$ is the reciprocal of the ultimate elongation $e_{\max }=\lambda_{\max }-\lambda_{\max }{ }^{-2}$ (repulsive interaction) and the parameter $a$ is related to local attractive interactions.

Accordingly, we obtain the following stress-strain relationship:

$$
\sigma=\kappa(1-\Theta)+\frac{\Re \Theta e}{1-b e}-a e^{2}
$$

where $\Theta=T / T_{p}, \kappa=\Delta H / V_{F}$ and $\mathfrak{R}=N k T_{p}$. This equation shows a phase transformation from unstrained (un-necked) to strained (necked) phase below a critical temperature. In addition, above the critical temperature the materials become too soft to be necked and instead deform homogeneously. Taking the first and second derivatives in equation (5) with respect to $e$ and setting them independently equal to zero, we obtain the critical strain $e^{*}=(3 b)^{-1}$, the critical reduced temperature $\Theta^{\star}=T^{*} / T_{p}=8 a /\left(27 b N k T_{p}\right)$ where $T^{*}$ is the critical temperature, and the critical stress $\sigma^{\star}=a /\left(27 b^{2}\right)$. Then, the original constitutive equation can be reduced by normalizing the original variables $e, \Theta$ and $\sigma_{e}$ in terms of this critical point to:

$$
\Sigma=\Lambda\left(1-\frac{T *}{T_{p}} \tau\right)+\frac{8 \tau \xi}{3-\xi}-3 \xi^{2}
$$

where the reduced stress, $\Sigma=\sigma / \sigma^{*}$, the reduced temperature, $\tau=\Theta / \Theta^{*}$, the reduced strain, $\xi=e / e^{*}$, and the coefficient of plastic component, $\Lambda=8 \kappa /\left(3 N k T^{*} e^{*}\right)$. Equation (6) requires the condition $0 \leqslant \xi \leqslant 3$. As shown in the previous paper, ${ }^{17}$ equation (6) can be reduced to the cusp catastrophe equation. ${ }^{27}$ A three-dimensional plot of equation (6) is shown in Figure 2. The maximum point of the reduced stress $\Sigma$ is a bifurcation point. When the extent of deformation reaches the bifurcation point, the deformation point jumps horizontally to the cross point of the horizontal line and the curve of equation (6). This sudden process leads to a highly strained or ordered phase. However, this sudden structural transformation cannot reach the whole specimen so that the highly oriented region is confined to a very local portion of the specimen, and forms a seed for necking.

\section{RESULTS AND DISCUSSION}

Figure 3 shows the temperature dependence of the stress-strain curves measured at an elongation speed of $0.1 \mathrm{~mm} \mathrm{~s}^{-1}$. The stress-strain curves clearly show the existence of double yield points. A sharp first yield point is observed at lower temperatures below the $\alpha$-relaxation of the motion of chain units within the crystalline phase, but as the temperature increases, this gradually becomes less pronounced and a broader second yield seems to dominate above $75^{\circ} \mathrm{C}$. As previously reported, ${ }^{9}$ photographs of deformed specimens showed that the first yield point is not associated with necking, and that specimens are homogeneously deformed up to around the first yield point. ${ }^{9}$ Neck precursors are stabilized at the second yield point. Similar photos were previously published by Brooks et al. ${ }^{10}$ They suggest that a load maximum at the first yield point is intrinsic and is associated with a strain-softening process, whereas a second yield peak is geometric.

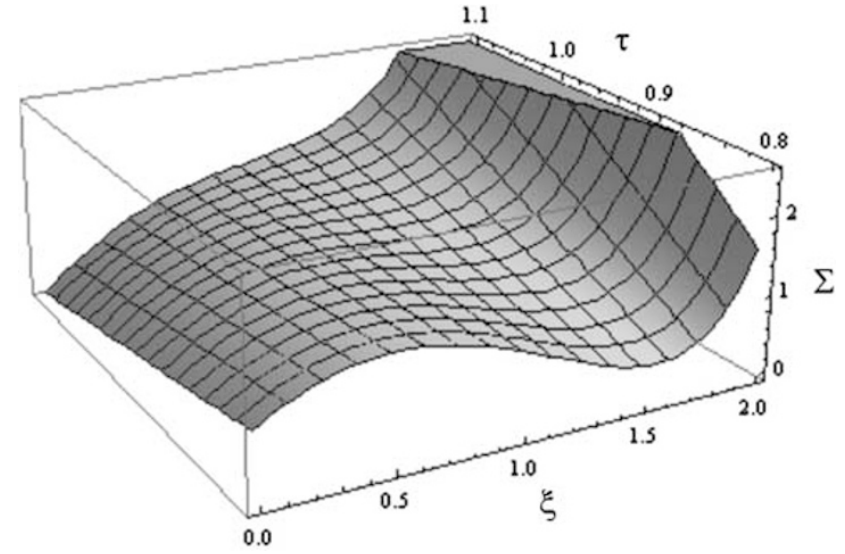

Figure 2 Reduced stress $\Sigma$ plotted against reduced strain $\xi$ and reduced temperature $\tau$. The plastic component $A$ is taken to be $2, T_{p}=361 \mathrm{~K}$ and $T^{*}=335 \mathrm{~K}$. A full color version of this figure is available at Polymer Journal online.

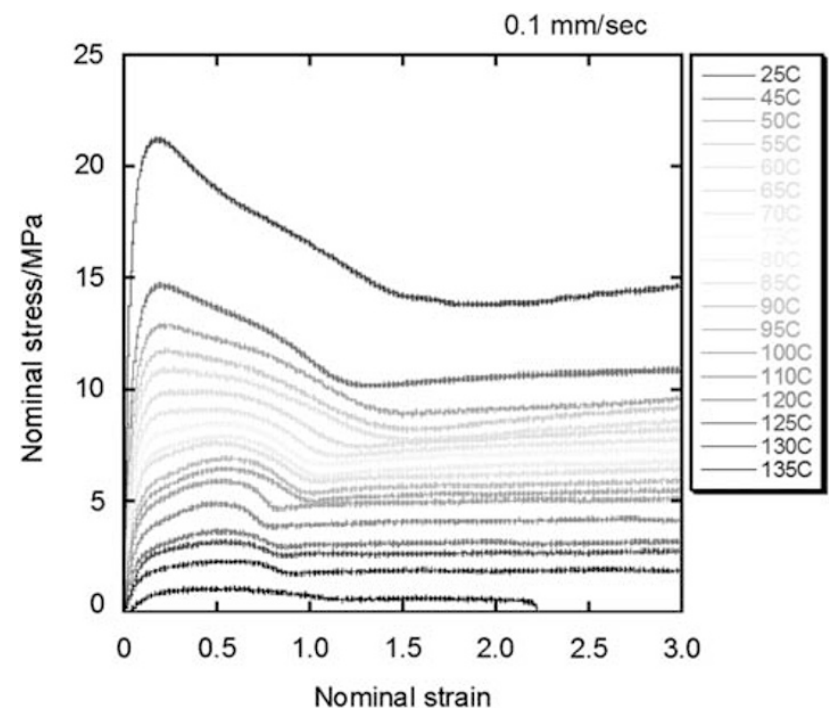

Figure 3 Temperature dependence of stress-strain curves of HDPE measured at an elongation speed of $0.1 \mathrm{~mm} \mathrm{~s}^{-1}$. A full color version of this figure is available at Polymer Journal online.

We found that the neck initiates at the inflexion point between the first yield peak and the second yield shoulder, producing a shallow shoulder in the specimen. In the current work, the initiation point of necking is taken as the inflexion point.

As shown in Figure 4, an increase in the elongation speed enhances the overall stress level of the yielding and necking zones within the stress-strain curves measured at $25^{\circ} \mathrm{C}$. The flow stress values in the necking zone and the natural draw ratios are plotted against the temperature in Figure 5. The flow stress linearly decreases to zero with two break points as the temperature increases. It is interesting to note that the intercept on the temperature axis is $415 \mathrm{~K}$, which is identical to the equilibrium melting temperature of $\mathrm{PE}$. The break points correspond to the $\alpha_{1}$ (about $70{ }^{\circ} \mathrm{C}$ ) and $\alpha_{2}$ (about $120^{\circ} \mathrm{C}$ ) viscoelastic relaxations in the dynamic mechanical loss modulus spectrum (see Figure 6), where the $\alpha_{1}$ relaxation is attributed to an intralamellar slip process (or grain boundary phenomenon) and/or motion in the intercrystalline region, and the $\alpha_{2}$ relaxation is attributed to an intracrystalline chain motion involving a translational motion of chain segments along the $c$ axis within the crystal lattice. ${ }^{28}$ 


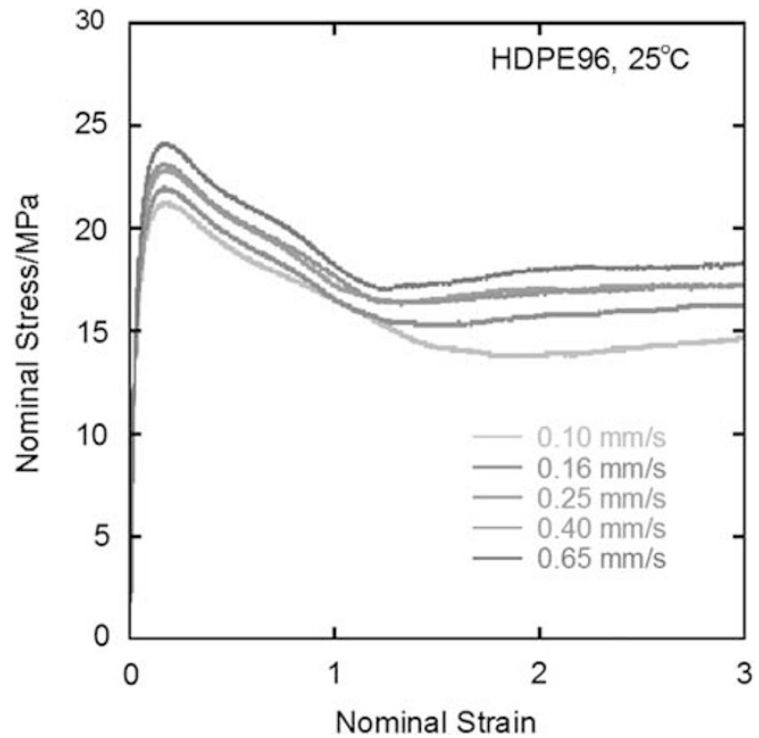

Figure 4 Elongation speed dependence of stress-strain curves of HDPE measured at $25^{\circ} \mathrm{C}$. A full color version of this figure is available at Polymer Journal online.

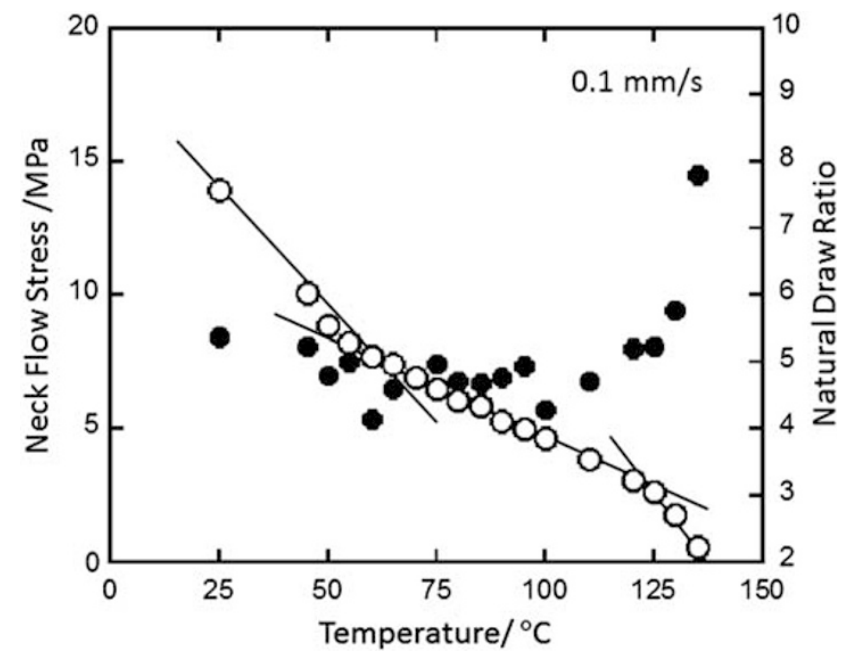

Figure 5 Temperature dependence of neck flow stress (open circle) and natural draw ratio (closed circle) measured at an elongation speed of $0.1 \mathrm{~mm} \mathrm{~s}^{-1}$.

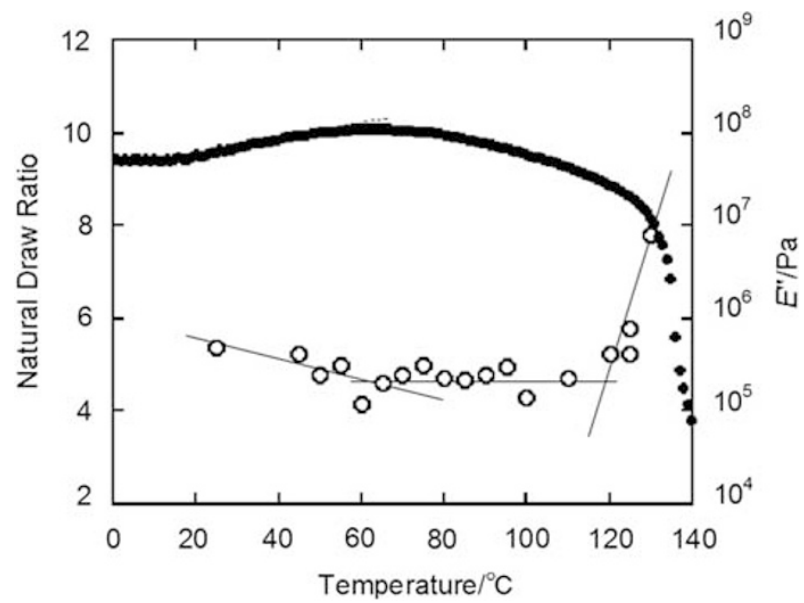

Figure 6 Comparison of loss modulus (solid lines) and natural draw ratio (open circle).
These results also suggest that the neck flow stress is associated with the plastic deformation of the crystalline phase. In addition, the experimental values of flow stress become independent of the elongation speed above about $70^{\circ} \mathrm{C}$ and approach zero at the melting point. The natural draw ratio is almost constant or slightly decreases with increasing temperature below $120^{\circ} \mathrm{C}$, whereas it rapidly increases above $120^{\circ} \mathrm{C}$. This indicates that the necking behavior above $120^{\circ} \mathrm{C}$ is similar to the melt-spinning necking behavior because viscous flow predominantly occurs above $120^{\circ} \mathrm{C}$. Thus, there are three different deformation modes: cold drawing (below $\alpha_{1}$ relaxation), intermediate drawing (between $\alpha_{1}$ and $\alpha_{2}$ relaxations) and viscoelastic drawing (above $\alpha_{2}$ relaxation). It follows that the cold-drawing characteristics take place below about $70^{\circ} \mathrm{C}$ or in the pre- $\alpha$ relaxation regions. Therefore, the critical point of the cold-drawing process is likely to be the $\alpha_{1}$-relaxation temperature before the onset of the activation of

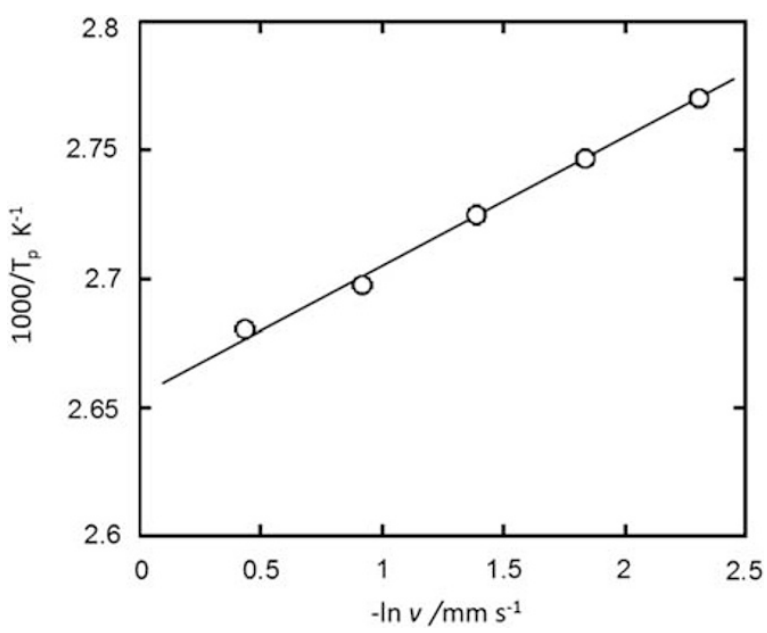

Figure 7 Elongation speed dependence of $T_{\mathrm{p}}$.

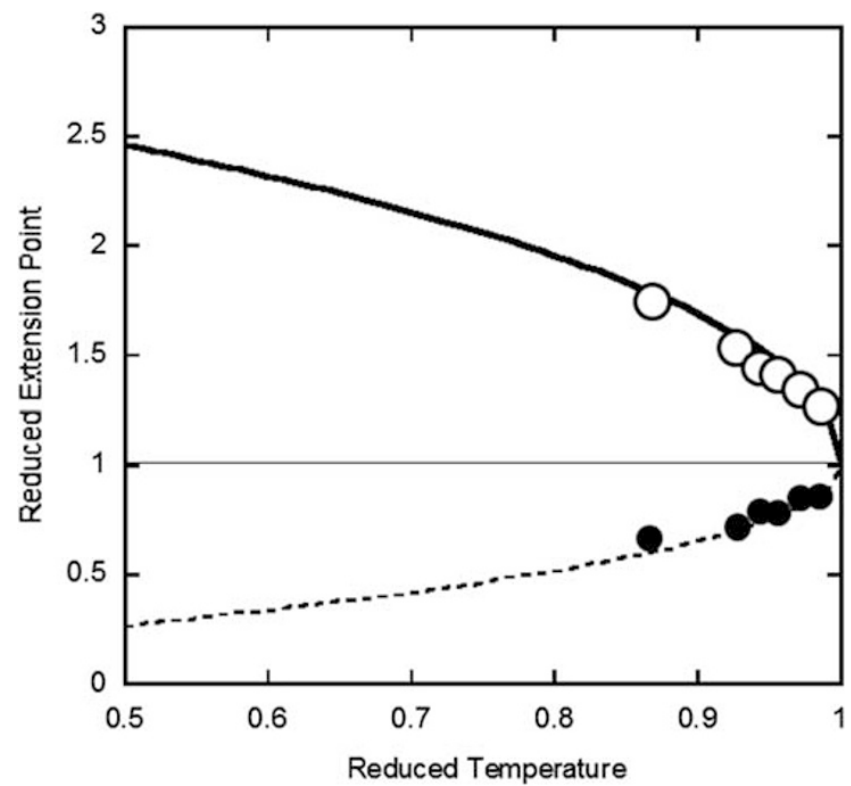

Figure 8 Theoretical and experimental values of the reduced first yield extension $\xi_{1}$ (dot line and closed circle) and the reduced extension at neck initiation $\xi_{\text {int }}$ (solid line and open circle). 


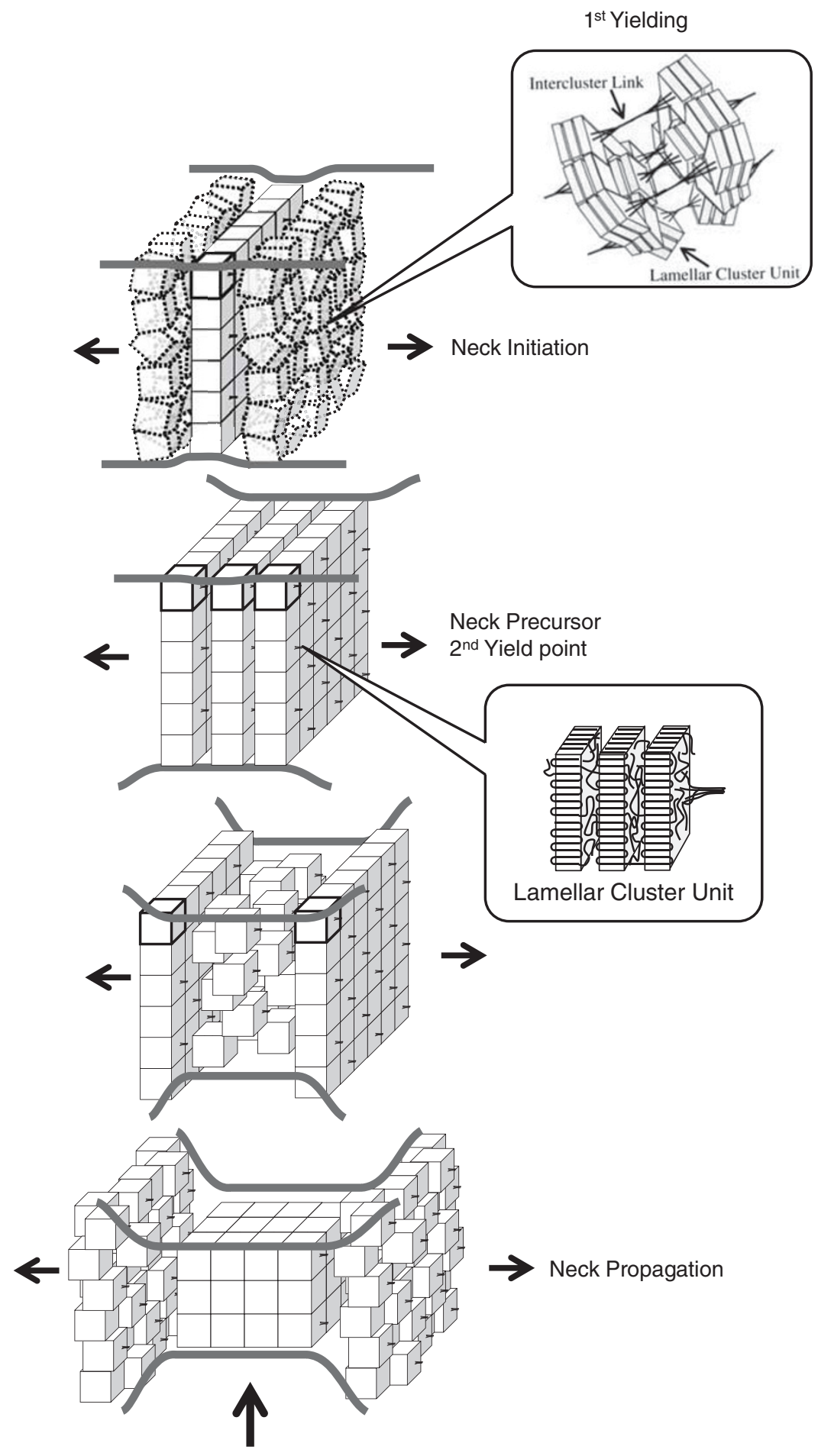

Natural draw state

Figure 9 Sketch of the necking process just after the first yield point.

chain mobility in the crystalline phase. Here, we set $T^{*}=70{ }^{\circ} \mathrm{C}$ because the termination temperature of cold drawing can be considered to be the critical temperature $T^{*}$.

The temperature dependence of flow stress below $70{ }^{\circ} \mathrm{C}$ can be fitted with equation (2) as shown in Figure 5 , and the temperature $T_{p}$ can be determined from the intercept of the line on the temperature axis. Similar results were obtained for other elongation speeds.
The reciprocal values of $T_{p}$ measured at other elongation speeds were found to be inversely proportional to the natural logarithm of the extension speed values as predicted by equation (3) (see Figure 7) where the elongation speed is assumed to be proportional to the strain rate $\dot{\varepsilon}$. The flow stress values resulting from the plastic flow of structural units involving crystalline parts approach zero as the temperature approaches $T_{p}$. 
The maximum point of the reduced stress $\Sigma$ in equation (6) corresponds to the onset of the necking process, that is, the reduced strain $\xi_{1}$ at the maximum point is given by the first yield strain $e_{1 \mathrm{st}}$ $=\lambda_{1 \mathrm{st}}-1 / \lambda_{1 \mathrm{st}}{ }^{2}$ normalized by the critical $e^{\star}$, which is one-third of the maximum strain $e_{\max }$. The horizontal line passing the maximum point intersects the stress-strain relation, equation (6), giving the point of initiation of necking $\xi_{\text {int }}$, which is the dominant factor for the natural draw ratio. The strain at neck initiation was experimentally determined from the flexion point of the stress-strain curves between the first and second yield points; $e_{\text {int }}=\lambda_{\text {int }}-1 / \lambda_{\text {int }}{ }^{2}$. The maximum point $\xi_{1}$ and the intersection point $\xi_{\text {int }}$ can be analytically obtained from the derivative of equation (6) and the horizontal line passing the maximum point, giving:

$$
\xi_{1}=2\left(1-\cos \frac{2}{3} \phi\right), \quad \xi_{\text {int }}=3-4\left(1-\cos \frac{2}{3} \phi\right)
$$

where $\phi=\arcsin \sqrt{\tau}$. We have a simple relation $\xi_{\text {int }}=3-2 \xi_{1}$. $\xi_{\text {int }}$ monotonically decreases from 3 to 1 while $\xi_{1}$ monotonically increases from 0 to 1 with increasing reduced temperature $\tau \geqslant 0$, resulting in $\xi_{\text {int }}=\xi_{1}=1$ at the critical point $\tau=1$.

Taking the maximum extension $\lambda_{\max }$ to be the extension of the locally necked region at the second yield point, the value of $\lambda_{\max }$ can be given by $\left(\lambda_{2 \text { nd }}-\lambda_{1 \mathrm{st}}\right) /\left(\lambda_{\text {int }}-\lambda_{1 \mathrm{st}}\right)$. This value was found to be intrinsic, being almost independent of temperature and elongation speed. Consequently, we have $e^{\star}=0.73\left(\lambda_{\max }=1.3\right)$.

Using the critical values of $e^{\star}$ and $\mathrm{T}^{\star}$, we can experimentally obtain the $\xi_{1}$ and $\xi_{\text {int }}$. Figure 8 compares the reduced strains $\xi_{1}$ and $\xi_{\text {int }}$ as a function of the experimental reduced temperature $\tau$. The experimental $\xi_{1}$ and $\xi_{\text {int }}$ values are in reasonable agreement with the theoretical ones, suggesting that the necking is catastrophically initiated according to the present theory.

The lamellar clusters are fragmented into lamellar cluster units at the first yield point and the cluster units rotate toward the stretching direction. The units are visualized as identical cubic blocks with a single chain size. Further deformation leads to close packing of cluster units with a slight reduction of the cross-sectional area between the first and the second yield points. Several necking plates coalesce into a precursor of macroscopic necking at the second yield point, as shown in Figure 9. Consequently, the external load is uniformly applied normal to the necking layer. After the second yield point, the plane of a frontier layer collapses by sliding the blocks using the ICLs, and behaves as a unit for the necking process. The cubic cluster units within the layer are likely to rearrange to form a thermodynamically stable cuboid object. The length of the cuboid object determines the natural draw ratio. The continual collapse from one frontier wall into another corresponds to the neck propagation.

According to the scanning synchrotron small angle X-ray scattering studies of deformed HDPE, the necking leads to a smaller long spacing. ${ }^{29}$ As the cubic cluster units are assumed to be rigid, the current model has no ability to describe the changes in long spacing and lamellar thickness. The compression of cubic cluster units and deformation within the cubic units may need to be taken into account under close packing and rearrangement of cubic units during necking.

\section{CONCLUSIONS}

In the current work, we considered the mechanism of neck initiation. A catastrophic ordering caused by the coexistence of repulsive and attractive interactions was suggested to be the origin of initiation of necking.

Lamellar clusters are fragmented into lamellar cluster units at the yield point, and the cluster units rotate toward the stretching direction, where the cluster units act as a deformation unit under yielding and necking. Then the intercluster links (ICLs) support the external force, and their tractive forces act on the lamellar cluster surface, leading to fragmentation of lamellar clusters into cluster units at the first yield point. The rearrangement of ICLs decorated with cluster units gives the texture of necking, so the first yield point can be considered to be the creation of necking. The decorated ICLs are randomly and homogeneously dispersed in the spherulitic matrix just after the first yield point. A combination of the ordering process of ICLs (or attractive effect) and the exclusion process between the bulky cluster units (repulsive effect) leads to a van der Waals type of constitutive relation, analogous to the roles of parameters $a$ and $b$ in the conventional van der Waals gas. The coexistence of the repulsive and attractive effects causes a catastrophic phase transformation from unordered to ordered structure. This sudden bifurcation is the initiation of necking.

The condition leading to necking is the existence of networks with strong repulsive interactions. Therefore, amorphous and/or liquidcrystalline polymers with decorated ICL-like structures such as rigid amorphous components, crystallizable segments and rod-like molecules have the possibility of necking. Above $70^{\circ} \mathrm{C}$, the necking and yielding behaviors are much different from the cold-drawing behavior. In the $\alpha$-relaxation region between 70 and $120^{\circ} \mathrm{C}$, the lamellar cluster units are softened and the deformation of the units must be taken into account. Moreover, the deformation above $120^{\circ} \mathrm{C}$ may be regarded as the necking process of viscoelastic bars and fibrous materials because the differences in mechanical characteristics between the amorphous region and crystalline phase are obscure. These types of necking flow can be considered to be catastrophic phenomena. ${ }^{30}$ The catastrophe theory potentially will be a powerful new tool for providing physical insight into various mechanical instabilities of polymeric materials, such as stress-induced crystallization, neck flow of fibers and melt fracture.

1 Brooks, N. W., Duckett, R. A. \& Ward, I. M. Investigation into double yield points in polyethylene. Polymer 33, 1872-1880 (1992).

2 Carothers, W. H. \& Hill, W. J. Studies of polymerization and ring formation. XV. Artificial fibers from synthetic linear condensation superpolymers. J. Am. Chem. Soc 54, 1579 (1932).

3 Séguéla, R. \& Darras, O. Phenomenological aspects of the double yield of polyethylene and related copolymers under tensile loading. J. Mater. Sci. 29, 5342-5352 (1994).

4 Séguéla, R. On the natural draw ratio of semi-crystalline polymers: review of the mechanical, physical and molecular aspects. Macromol. Mater. Eng. 292, 235-244 (2007).

5 Peterlin, A. Molecular model of drawing polyethylene and polypropylene. J. Mater. Sci. 6, 490-508 (1971).

6 Nitta, K. \& Takayanagi, M. Novel proposal of lamellar clustering process for elucidation of temsile yield behavior of linear polyethylenes. J. Macromol. Sci. Phys. B42, 107-126 (2003).

7 Takayanagi, M., Imada, K. \& Kajiyama, T. Mechanical properties and fine structure of drawn polymers. J. Polym. Sci. C 15, 263-281 (1966).

8 Keith, H. D., Padden, F. J. \& Vadimsky, R. G. Intercrystalline links in polyethylene crystallized from the melts. J. Polym. Sci. A-2 4, 267-281 (1966).

9 Takayanagi, M., Nitta, K. \& Kojima, O. Application of lamellar clustering theory to isotactic polypropylene and direct observation of lamellar cluster morphology by electron microscopy. J. Macromol. Sci-Phys. B B42, 1049-1059 (2003).

10 Kuriyagawa, M. \& Nitta, K. Structural explanation on natural draw ratio of metallocenecatalyzed high density polyethylene. Polymer 52, 3469-3477 (2011).

11 G'Sell, C., Aly-Helal, N. A. \& Jonas, J. J. Effect of stress triaxiality on neck propagation during the tensile stretching of solid polymers. J. Mater. Sci. 18, 1731-1742 (1983).

12 Huchingson, J. W. \& Neale, K. W. Neck propagation. J. Mech. Phys. Solid 31, 405-426 (1983).

13 Ericksen, J. J. Equilibrium of bars. J. Elasticity 5, 191-201 (1975).

14 Heise, B., Kilian, H. G. \& Wulff, W. Deformation and microstructure in uniaxially stretched PE. Prog. Colloid Polym. Sci. 67, 143-147 (1980).

15 Killian, H.- G. Equation of state of real networks. Polymer 19, 209-217 (1981).

16 Kilian, H.- G. Small-strain elastic moduli of quasi-isotropic semicrystalline eutectoid copolymers and aspects of universality. Colloid Polym. Sci. 262, 374-380 (1984). 
17 Nitta, K. \& Takayanagi, M. Application of catastrophe theory to the neck-initiation of semicrystalline polymers induced by the intercluster links. Polymer J. 38, 757 (2006).

18 Nitta, K. \& Tanaka, A. Dynamic mechanical properties of metallocene catalyzed linear polyethylenes. Polymer 42, 1219 (2001).

19 G'Sell, C. \& Jonas, J. J. Yield and transient effects during the plastic deformation of solid polymers. J. Mater. Sci. 16, 1956-1974 (1981).

20 Haward, R. N. The application of a siblified model for the stress-strain curves of polymers. Polymer 28, 1485-1488 (1987)

21 Song, Y., Nitta, K. \& Nemoto, N. Molecular orientations and true stress-strain relationship in isotactic polypropylene film. Macromolecules 36, 8066-8073 (2003).

22 Eyring, H. Viscosity, plasticity, and diffusion as examples of absolute reaction rates. J. Chem. Phys. 4, 283-291 (1936).

23 Nitta, K. \& Takayanagi, M. Role of tie molecules in the yielding deformation of isotactic polypropylene. J. Polym. Sci. B Polym. Phys. 37, 357-368 (1999).
24 Kanig, G. Direct observation of polyethylene fine structure and its changes during drawing and annealing. J. Cryst. Growth 48, 303-320 (1980).

25 Strobl, G. R. The Physics of Polymers 359 , (Springer-Verlag, Berlin, 1998).

26 Mark, J. E. in Physical Properties of Polymer 2nd edn, Ch. 1, 3-59 (ACS, Washington DC, 1993).

27 Poston, T. \& Stewart, I. Catastrophe Theory and Its Applications. (Pitman Pub., London, UK, 1978).

28 Kajiyama, T., Okada, T., Sakoda, A. \& Takayanagi, M. J. Macromol. Sci. Phys. B 7, 583 (1973).

29 Jiang, Z., Tnag, Y., Men, Y., Enderle, H- F., Lilge, D., Roth, S. V., Gehrke, R. \& Rieger, J. Structural evolution of tensile-deformed high-density polytheylene during annealing: scanning synchrotron small-angle X-ray scattering study. Macromolecules 40, 7263-7269 (2007).

30 Ohkoshi, Y., Watanabe, J., Okumura, W., Gotoh, Y. \& Nagura, M. Catastrophic neck-flow transition during laser-heated drawing of poly(ethylene terephthalate) fiber. Sen' Gakkaishi 58, 182-184 (2002). 